\title{
Broad- and narrowband visible imaging of comet 9P/Tempel 1 at ESO around the time of the Deep Impact event ${ }^{\star}$
}

\author{
H. Boehnhardt ${ }^{1}$, E. Pompei ${ }^{2}$, G. P. Tozzi ${ }^{3}$, O. Hainaut ${ }^{2}$, N. Ageorges ${ }^{2}$, S. Bagnulo ${ }^{2}$ \\ L. Barrera ${ }^{4}$, T. Bonev ${ }^{5}$, H. U. Käufl ${ }^{6}$, F. Kerber ${ }^{6}$, G. LoCurto ${ }^{2}$, O. Marco ${ }^{2}$, E. Pantin ${ }^{7}$, H. Rauer ${ }^{8}$, I. Saviane ${ }^{2}$, \\ F. Selman ${ }^{2}$, C. Sterken ${ }^{9}$, and M. Weiler ${ }^{8}$ \\ 1 Max-Planck Institute for Solar System Research, Max-Planck-Str. 2, 37191 Katlenburg-Lindau, Germany \\ e-mail: boehnhardt@mps.mpg.de \\ 2 European Southern Observatory, Alonso de Cordova 3107, Vitacura, Santiago de Chile \\ 3 INAF Arcetri Observatory, Largo E. Fermi 5, 50125 Firenze, Italy \\ ${ }^{4}$ Universidad Metropolitana de Ciencias de la Educacion, Av. J.P. Alessandri 774, Nunoa, Santiago de Chile \\ 5 Bulgarian Academy of Sciences, Tsarigradsko chaussee 72, 1784 Sofia, Bulgaria \\ ${ }^{6}$ European Southern Observatory, Karl-Schwarzschild-Str.2, 85748 Garching, Germany \\ 7 Commissariat Energie Atomique, 91191 Gif-sur-Yvette, France \\ 8 Deutsche Luft- und Raumfahrt Agentur, Rutherfordstr. 2, 12489 Berlin-Adlershof, Germany \\ 9 Vrije Universitet Brussel Pleinlaan 2, 1050 Brussels, Belgium
}

Received 16 October 2006 / Accepted 18 May 2007

ABSTRACT

\begin{abstract}
Context. On 4 July 2005 at 05:52UT the impactor of NASA's DeepImpact (DI) mission exploded at comet 9P/Tempel 1. The ejecta material of the impact expanded into the coma that is produced by normal cometary activity. The La Silla and Paranal sites of the European Southern Observatory ESO in Chile participated in the world-wide campaign to observe this event.

Aims. The gas and dust content of the cometary coma is observed around the time of the DI event to identify signatures of the impact, like changes in the gas production $\left(\mathrm{CN}, \mathrm{C}_{3}, \mathrm{C}_{2}, \mathrm{NH}_{2}\right.$ and $\left.\mathrm{Na}\right)$ and in the dust properties. The study also describes the normal activity pattern in the coma and the expanding ejecta cloud.

Methods. The gas production rates and the dust reddening slope are measured in images obtained through narrowband cometary filters. The ejecta cloud and the features of normal cometary activity, as imaged in narrow- and broadband filter images, are studied with respect to geometry, intensity, and persistence.

Results. The production of $\mathrm{CN}, \mathrm{C}_{3}$, and $\mathrm{C}_{2}$ gas by the nucleus is at similar level on 3 July and 9+10 July 2005. The mixing ratios of these gases are in the range of those for "typical" comets. $\mathrm{NH}_{2}$ and $\mathrm{Na}$ gas are not detected above the dust continuum flux in the coma. The reddening slope of the dust continuum changes from a value of $9 \% / 100 \mathrm{~nm}$, constant throughout $20000 \mathrm{~km}$ distance around the nucleus, on 3 July to about 19-20\%/100 nm on 9+10 July 2005. An increase of the slope with radial distance is found. Both changes might be due to the presence of dust from the ejecta cloud. The dust coma of the comet showed a porcupine pattern of 9 coma jets before impact which remained intact after impact. It can be interpreted as being due to embedded fan structures produced by at least 4 active regions on the rotating nucleus. The ejecta cloud contains dust grains of likely absorbing material and possibly dielectric admixtures with solar radiation pressure factors $\beta$ of $0.2-1.9$ and ejection velocities of $160-370 \mathrm{~m} \mathrm{~s}^{-1}$. The ejecta cloud is optically thin $21 \mathrm{~h}$ after impact and shows a higher surface brightness and redder color in the sunward sector compared to the tailward side.
\end{abstract}

Key words. comets: individual: Comet 9P/Tempel 1

\section{1. "Deep Impact" at ESO La Silla and Paranal}

The Deep Impact project (DI), a combined impactor and fly-by mission to the Jupiter family comet 9P/Tempel 1 (9P), is the first man-made cratering experiment to probe a cometary nucleus (A'Hearn et al. 2005) with the aim to further conclude on physical properties of these small bodies in the solar system which are generally believed to be widely unmodified remnants from the formation period of the planetary system. The prime goal of the mission was to hit a comet with a $360 \mathrm{~kg}$ impactor at about $10 \mathrm{~km} \mathrm{~s}^{-1}$ velocity, to excavate a crater by the impact and to eject surface and, in particular, sub-surface material (gas and dust), that was not exposed to sunlight since incorporated in the nucleus, into the coma where it can be measured by the fly-by

$\star$ Based on observations performed at the ESO La Silla and Paranal Observatories in Chile (program 075.C-0583). spacecraft and other remote sensing instruments and telescopes on Earth and in space. Earth-based observations played a crucial role for the success of the mission since the post-impact visibility window of the impact region from the fly-by spacecraft was only a little more than $8 \mathrm{~min}$.

Here, we present results from imaging observations of comet 9P/Tempel 1 around the time of impact performed at the European Southern Observatory (ESO) Chile. We focus on the appearance of the overall coma produced by the normal activity of the comet and on production rates of coma gas around 4 July 2005. The results on the dust component of the DI ejecta cloud (Tozzi et al. 2007; Bonev et al. 2007) and on the thermal and polarimetric properties (papers in preparation) of the dust in comet 9P/Tempel 1, obtained at ESO, are described elsewhere. Results from the spectroscopic observations of the gas phase at ESO are presented in papers by Rauer et al. (2006) and Jehin et al. (2006). 
Table 1. Instrument filter setup and filter properties during the DI observations at ESO. The broadband filters are annotated following the standard photometry designation. Note that $R_{\text {special }}$ is a modified $R$ Bessell filter - see http://www. eso.org/instruments/fors $1 /$ for further details. The names of the narrowband gas filters $\left(\mathrm{CN}, \mathrm{C}_{2}, \mathrm{C}_{3}, \mathrm{NH}_{2}\right)$ refer to the respective gaseous species that emit light in the filter passband. Dust continuum filters are labeled BC for "blue continuum" and RC for "red continuum", a narrowband OII filter was used to measure the dust continuum in the UV. A special Na filter was installed for imaging of the comet in the wavelength range of the sodium D lines.

\begin{tabular}{llcccc}
\hline \hline $\begin{array}{l}\text { Observatory/ } \\
\text { telescope/ } \\
\text { instrument }\end{array}$ & Light path & $\begin{array}{c}\text { Filter } \\
\text { name }\end{array}$ & $\begin{array}{c}\text { Central } \\
\text { wavelength } \\
(\mathrm{nm})\end{array}$ & $\begin{array}{c}\text { Band } \\
\text { width } \\
(\mathrm{nm})\end{array}$ & $\begin{array}{c}\text { Max. } \\
\text { transmission } \\
\%\end{array}$ \\
\hline VLT/UT1/FORS2 & parallel & $B$ & 429 & 88.0 & 64.0 \\
& beam & $V$ & 554 & 111.5 & 88.0 \\
& & $R_{\text {special }}$ & 655 & 165.0 & 95.0 \\
& $I$ & 768 & 138.0 & 96.0 \\
\hline LSO/NTT/EMMI & blue arm - & $\mathrm{OII}$ & 372.550 & 6.882 & 34.2 \\
& convergent & $\mathrm{CN}$ & 385.971 & 5.013 & 42.6 \\
& beam & $\mathrm{C}_{3}$ & 405.229 & 7.538 & 58.8 \\
& red arm - & $\mathrm{BC}$ & 442.164 & 3.670 & 67.3 \\
& parallel & $\mathrm{C}_{2}$ & 511.069 & 12.278 & 85.7 \\
& beam & $\mathrm{Na}$ & 589.347 & 2.300 & 59.2 \\
& & $\mathrm{NH}_{2}$ & 662.543 & 3.455 & 85.2 \\
& & $\mathrm{RC}$ & 683.816 & 8.089 & 86.0 \\
\hline
\end{tabular}

The observations mentioned above were performed at the two optical observatories of ESO in Chile in the context of the World Observatory campaign to monitor and explore the phenomena generated by the DeepImpact mission at comet 9P/Tempel 1. An overview of the scientific goals and performance of the observations at ESO as a whole is given by Käufl et al. (2005) and further documented in the outreach section of the ESO web page (http://www.eso.org), while the worldwide campaign and first results of it are summarized in Meech et al. (2005).

\section{Observations and data reduction}

In this paper we present results from broadband imaging of 9P as well as from narrowband imaging using special cometary filters.

Observing equipment for broadband imaging: the Bessell BVRI filter imaging of 9P/Tempel 1 was performed at the ESO Paranal Observatory using the Very Large Telescope (VLT) unit telescope Antu (also called UT1) and the FORS2 instrument. The VLT UTs are basically identical telescopes of $8.2 \mathrm{~m}$ aperture, equipped with active optics, and provide - besides the combined interferometric focus - three single instrument focal stations per telescope. At UT1, FORS2 is mounted to the Cassegrain focus of the telescope. FORS2 is a multi-mode focal-reducer-type instrument for the visible and near-UV wavelength range that allows imaging and spectroscopic observations in different configurations and setups (for instance, different filters for imaging). The instrument is equipped with a two-chip $2 \times 2048 \times 4096$ pixels CCD detector assembly of 0.125 arcsec pixel resolution and $6.8 \times 6.8$ arcmin field of view. During our observations $2 \times 2$ pixel binning of the detectors was applied (effective pixel resolution $=0.25$ arcsec). The $B V R I$ broadband filters are installed in the parallel beam of the instrument (see Table 1). For technical details see the ESO web page: http://www.eso.org/instruments/ for VLT telescopes and instrumentation in general and http: //www . eso.org/instruments/fors1/for the FORS2 instrument in particular. The schedule and setup used for the 9P/Tempel 1 broadband imaging during 2-10 July 2005, as well as the environmental conditions are listed in Table 2.

Observing equipment for narrowband imaging: the narrowband imaging of the comet was obtained at the ESO
La Silla Observatory (LSO) using the New Technology Telescope (NTT) and the EMMI instrument. The NTT is a $3.5 \mathrm{~m}$ telescope with two main Nasmyth focal stations. Like the two FORS instruments at the VLT, EMMI is a multi-mode focal-reducer-type instruments for imaging and spectroscopic observations in the visible wavelength range. The instrument has two arms, one each tuned for the blue/UV and for the red wavelength range, respectively. The blue arm has a single-chip $1024 \times 1024$ pixels CCD detector of 0.37 arcsec pixel resolution and $6.2 \times 6.2$ arcmin field of view. The red arm carries a two-chips $2 \times 2048 \times 4096$ pixels CCD detector assembly of 0.166 arcsec pixel resolution and $9.9 \times 9.1$ arcmin field of view. $1 \times 1$ pixel binning was used in the blue arm (effective pixel resolution $=0.37$ arcsec) and $2 \times 2$ pixels bining in the red arm (effective pixel resolution $=0.33$ arcsec). For technical details see the ESO web page: http://www.ls.eso.org/ lasilla/sciops/ntt/index.html for the telescope and instrumentation and http://www.ls.eso.org/lasilla/ sciops/ntt/emmi/index.html for the EMMI instrument. The narrowband filters used for the comet observations in the EMMI blue and red arm, are listed in Table 1. Schedule, instrument setup and environmental conditions of the 9P/Tempel 1 narrowband imaging during 2-10 July 2005 are given in Table 2.

Observations: all comet observations were conducted in visitor mode with experienced comet observers being present at the telescopes and directing the execution of the imaging exposures. Given the complexity of the overall multi-mode observing schedule of the DI event at ESO and the variable sky conditions at the La Silla observatory during 2-10 July 2005 in particular, only low priority was set to achieve broad- and narrowband imaging of the comet simultaneously from both observatories. In each night a series of exposures (4-6) of the comet were taken per filter. The exposure series were exposed within a time interval of 10 to $30 \mathrm{~min}$. Small amplitude jitter offsets of the telescope were applied between individual filter exposures in order to allow full-field image reconstruction of the blind gap in the detector arrays and the removal of CCD artifacts. For calibration purposes twilight flatfield images for each filter and bias frames were taken during several nights of the observing runs. With FORS2 photometric standard stars from the Landolt list (1992) were measured in BVRI filters at different airmass during each night when the coma was imaged. For the flux 
Table 2. Log of the DI broad- and narrowband observations at the ESO observatories in Chile. For ESO Chile the observing window of the comet started around 23:30 UT (Sun about $18 \mathrm{deg}$ below horizon) and lasted until 04:20 UT (comet at 20 deg elevation). The following notations are used for the sky conditions: CLR = clear sky; THN = thin cirrus clouds; COUT=clouded out. The seeing values are measured by a dedicated seeing-monitoring telescope on-site.

\begin{tabular}{|c|c|c|c|}
\hline $\begin{array}{l}\text { Date } \\
\text { (UT) }\end{array}$ & $\begin{array}{l}\text { Observatory/ } \\
\text { instrument/ }\end{array}$ & $\begin{array}{l}\text { Filters } \\
\text { (Table 1) }\end{array}$ & $\begin{array}{l}\text { Sky conditions/ } \\
\text { seeing (arcsec) }\end{array}$ \\
\hline \multirow{2}{*}{$2-3 / 7 / 2006$} & VLT/FORS2 & - & CLR/0.6-0.9 \\
\hline & LSO/EMMI & OII, CN, $\mathrm{C}_{3}, \mathrm{BC}, \mathrm{C}_{2}, \mathrm{Na}, \mathrm{NH}_{2}, \mathrm{RC}$ & CLR-THN/1.5-2.0 \\
\hline \multirow[t]{2}{*}{$3-4 / 7 / 2006$} & VLT/FORS2 & $B, V, R_{\text {special }}, I$ & CLR/0.5-0.8 \\
\hline & LSO/EMMI & $\mathrm{CN}$ & $\mathrm{THN} / 1.0-2.0$ \\
\hline \multirow[t]{2}{*}{$4-5 / 7 / 2006$} & VLT/FORS2 & $B, V, R_{\text {special }}, I$ & CLR/0.6-1.0 \\
\hline & LSO/EMMI & $\mathrm{C}_{2}, \mathrm{Na}, \mathrm{RC}$ & CLR/1.0-2.0 \\
\hline \multirow[t]{2}{*}{$5-6 / 7 / 2006$} & VLT/FORS2 & $B, V, R_{\text {special }}, I$ & CLR/0.5-0.8 \\
\hline & LSO/EMMI & - & COUT \\
\hline \multirow[t]{2}{*}{$6-7 / 7 / 2006$} & VLT/FORS2 & $B, V, R_{\text {special }}, I$ & CLR/0.4-0.9 \\
\hline & LSO/EMMI & - & THN/1.0 \\
\hline \multirow[t]{2}{*}{$7-8 / 7 / 2006$} & VLT/FORS2 & $B, V, R_{\text {special }}, I$ & CLR/0.5-0.9 \\
\hline & LSO/EMMI & OII, CN, $\mathrm{C}_{3}, \mathrm{BC}, \mathrm{C}_{2}, \mathrm{Na}, \mathrm{NH}_{2}, \mathrm{RC}$ & THN/0.6-1.6 \\
\hline \multirow[t]{2}{*}{$8-9 / 7 / 2006$} & VLT/FORS2 & $B, V, R_{\text {special }}, I$ & CLR/0.7-1.3 \\
\hline & LSO/EMMI & $\mathrm{CN}, \mathrm{BC}, \mathrm{C}_{2}, \mathrm{RC}$ & CLR/0.5-1.0 \\
\hline \multirow[t]{2}{*}{$9-10 / 7 / 2006$} & VLT/FORS2 & $B, V, R_{\text {special }}, I$ & CLR/0.6-1.1 \\
\hline & LSO/EMMI & OII, $\mathrm{CN}, \mathrm{C}_{3}, \mathrm{BC}, \mathrm{C}_{2}, \mathrm{Na}, \mathrm{NH}_{2}, \mathrm{RC}$ & CLR/0.4-1.0 \\
\hline
\end{tabular}

calibration of the narrowband imaging we used filter exposures of the spectrophotometric standard star LTT 6248 (Hamuy 1994), since pre-selected calibration stars (HD 120086 and HD 149363) from the list of International Halley Watch Standard Stars (A'Hearn \& Vanysek 1985) turned out not to be useful: the latter list relies in parts on different filter bandpasses and does not include all filters used for our observations of 9P; moreover, HD 120086 turned out to be classified as variable star.

Since the cometary coma was filling the field of view of the broadband exposures, separate sky background images were obtained for each filter - immediately after the comet imaging about 1 deg away from 9P/Tempel 1 in sunward direction. For narrowband imaging the background level at the edge of the detector mosaic was found to be representative for the sky flux close to the comet. During comet exposures the telescopes were tracking the moving target at the velocity of the comet. All other exposures used stellar or no tracking of the telescopes.

We like to note that $9 \mathrm{P} /$ Tempel 1 had set already for the Chilean observing sites by the time the impact happened at the comet. However, the ESO telescopes could monitor the comet appearance until a few hours before and as of $16 \mathrm{~h}$ after to 6 days after the event allowing to investigate changes due to DI for instance by comparison of the pre- and post-impact situations.

Basic data reduction: the calibration of the broadband images follows standard procedures for photometric calibrations of CCD exposures, i.e. bias subtraction and flatfield division (in each case applying median averaged frames from the available set of bias and filter flatfield exposures), exposure time division, sky background subtraction, extinction and zeropoint corrections using the standard star fields. At the end and for each filter, all calibrated images of a jitter sequence of a single observing night were aligned to the central brightness peak in the coma and median-averaged, which improves the signal-to-noise of the coma flux and widely removes cosmic rays, trails from background stars as well as the inter-chip gaps of the CCD detectors of FORS2.

The data reduction of the narrowband images followed the procedure described for the broadband data except for the flux calibration: here, the flux level of the spectrophotometric standard star LTT 6248 was determined by multiplying the published flux spectrum of the star (Hamuy 1994) and the respective filter transmission curve measured for each filter in the lab. After proper extinction corrections using the table of mean extinction versus wavelength for LSO, again multiplied with the transmission curve of each filter, it was possible to calibrate the measured counts of the comet exposures in absolute flux units. At the end a median averaged composite of all exposures taken per filter and night, was computed following the procedure described for the broadband imaging reduction.

Coma structure enhancement: all combined images are further processed to enhance coma structures. Different methods are applied - in the same way to all available median averaged images and in many cases also to the single exposures. These enhancement methods are: (1) radial renormalization (described by A'Hearn et al. 1986); (2) radial and azimuthal masking (described by Schwarz et al. 1997); (3) masking with a coma reference image from another night (both images are subtracted from or divided by each other); (4) adaptive Laplace filtering (described by Boehnhardt \& Birkle 1994) and (5) wavelet filtering (described by Murtagh et al. 1993); and (6) simple unsharp masking (i.e. division or subtraction of a smoothed version of the original by the original image). The first three methods require accurate image centering which, if not properly done, can introduce artificial structures in the immediate neighbourhood of the pixel used for centering. The other methods are not affected by centering problems. All methods have strengths and weaknesses that can depend on the coma structure geometry, the signal-tonoise and the star background in the images or on a variety of other factors.

Hence, for the identification of coma structures in the processed images various verification criteria are applied, i.e. (1) the detection in the combined and/or single images by different methods; (2) the recognition of a deviating isophote pattern in the respective pixel area of the unprocessed image and (3) the detection of related coma structures in images of different exposure series (i.e. taken through different filters) and/or in different observing nights.

In the case of our 9P/Tempel 1 images, the most detailed results for the overall characterization of structures inherent in the coma are achieved - and hence listed below - using the adaptive Laplace and wavelet filtering methods. We also make very much use of images enhanced through radial renormalization since this 
method - although less sensitive to very weak coma structures shows the large-scale geometry of possibly related coma structures, i.e. of coma structures that might originate from the same region on the nucleus. For the enhancement of the DI ejecta cloud, method 3 above gave very useful results since it removed best the general coma background of the comet. In the latter case, we used filter exposures obtained during nights $2-3$ and 3-4 July 2005 as reference frames for narrowband and broadband images, respectively. Images processed by methods 2 and 6 did not reveal additional coma structures compared to the other ones.

In order to characterize identified coma structures, we determined some geometric information (like its near-nucleus position angle, extension, curvature). This was measured in the respective enhanced image or in a polar coordinate representation of it (using the central brightness peak in the coma and the North direction as zero points for the radial distance and position angle, respectively).

Gas production rates: the gas production rates are obtained using flux measurements from the combined calibrated images taken with narrowband filters. The two-dimensional dust continuum flux in the gas emission bands is interpolated using a dust continuum filter image (in BC or RC) of the night and the reddening slope of the coma dust continuum. The latter parameter is obtained - in the first place as two-dimensional image through a linear and pixelwise fit of the fluxes in the BC and RC dust continuum filters. Since these reddening slope images vary only moderately (few percent/100 nm) across the coma, and in order to reduced the pixel noise in the resulting gas flux image, we applied constant reddening factors for the dust continuum interpolation of the gas emission band images (see Table 5). If only a single dust continuum image was available in a particular night, a constant reddening of $10 \% / 100 \mathrm{~nm}$ is assumed. This value agrees with the dust reddening slope measured on 2-3 July 2005 (when our data have the best signal-to-noise level SNR) and it furthermore is compatible with the rather constant dust reddening as found for the comet by the ROSETTA OSIRIS team within a few days pre- and post-impact (Küppers et al. 2005). The interpolated dust continuum flux is subtracted from the respective combined calibrated gas filter image. The resulting frame is median filtered using a $15 \times 15$ pixels filter width for SNR improvement. In the final image the gas emission flux is measured in concentric apertures of 5000, 10000,15000 and $20000 \mathrm{~km}$ radius. Note: measurements of smaller and larger apertures are considered unreliable, since they may be affected - for smaller distances - by seeing variations and image decentering or - for larger distances - by insufficient SNR in the images used for the dust continuum interpolation. If after continuum subtraction, gas emission flux is detected, the resulting flux values $F$ are used to determine the number $M$ of gas molecules emitting in the measurement aperture

$M=\frac{4 \pi \Delta^{2} \times 2 r^{2}}{g} F$

where $r$ and $\Delta$ are the comet distances to the Sun and to Earth, respectively, and $g$ is the fluorescence efficiency of the respective gas molecule at $1 \mathrm{AU}$ from the Sun.

Using $M$, the standard Haser model (Haser 1957) and the necessary model parameters (photodissociation scalelengths for the mother and daughter species, gas expansion velocity $\left(1 \mathrm{~km} \mathrm{~s}^{-1}\right.$ ), fluorescence efficiency $g$ ) the molecule production rate $Q$ can be estimated. For the above mentioned model parameters we applied the values given by A'Hearn et al. (1995) including the radial velocity dependent $\mathrm{g}$-factor for the $\mathrm{CN}$ molecule as calculated by Schleicher (1982). The resulting $Q$ values for the four apertures measured are averaged for each molecule and each night.

The Haser model assumes continuous and isotropic outflow of the gas, requirements that are certainly not applicable for the gas content produced by the DI event. However, the results, when comparing the pre- and post-impact situation, should still be indicative for changes in gas production, either due to the impact event or due to the natural variability of the comet (rotational changes, outbursts).

\section{Coma structures}

Several structures are identified in the enhanced images of the cometary coma around the time of the DI event. Before the impact event the coma showed the porcupine geometry, described by Lara et al. (2006) and interpreted as embedded fan coma. After impact, this structures is overlapped in our images by the DI ejecta cloud.

Nature of the coma structures: the coma features described here are seen in all broadband filter images and many of them are seen in the narrowband (gas and dust) filter images as well. This indicates that the coma structures seen during 2-10 July 2005 are dominated by dust. Only the $\mathrm{CN}$ filter images gave indications for gaseous coma structures to be present in the coma.

Coma structures from normal activity: in the Laplacefiltered images in total 9 straight or curved extended coma structures are identified. They are caused by normal activity of the comet. The jet-like features are best seen in the BVRI images (since the signal-to-noise is highest), but also apparent in narrowband filter exposures of the comet. We annotate them A, B, C, D, E, F, G, H, I in Fig. 1. Table 3 lists global geometric properties of the jets as measured from the available images. The position angle and shape of the features did not vary with time except for structure B, a straight linear jet in anti-solar direction. This feature is in the region of the dust and ion tails. Its variability (for instance it was brighter than structure E on 4 July, but fainter on 5 July) may be related to different mass loading into the tailward coma. The projected width of B is rather wide $(>6000 \mathrm{~km})$ and its uniform appearance makes an interpretation as ionic phenomenon unlikely. Moreover, there is no counterpart of structure $\mathrm{B}$ in narrowband $\mathrm{CN}$ images although all other strong coma structures of 9P/Tempel 1 (for instance A, D-F, G) do show CN emission structures in our images around the time of the impact event (Fig. 2). There are also no indications from narrowband ion filter exposures nor from spectroscopy that comet 9P/Tempel 1 had a rich plasma environment. The other straight and curved jets - as well as the overall coma brightness - remained constant in intensity. This is demonstrated in the masking enhancement technique (method 3 described briefly in Sect. 2) which results in a close to uniform background surrounding the ejecta cloud when subtracting (or dividing) a pre-impact from (or by) a postimpact image of the comet taken through the same filter. The $\mathrm{CN}$ emission is particularly strong for the sunward jet $\mathrm{G}$, second strongest is $\mathrm{A}$ and the fan area D-F.

The radially renormalized images (method 1 in Sect. 2) show only the narrow structure $G$ and a rather diffuse wide sector extending from South to East that possibly represents structures D, E, F (see Fig. 3). This feature suggests the presence of a coma fan that emits dust into the coma along a conical surface while the nucleus is rotating (Sekanina 1987). The other coma structures identified in the Laplace-filtered images are at best only marginally detected - if at all. It is noteworthy that all structures, originating from normal activity of the comet, are detectable in 

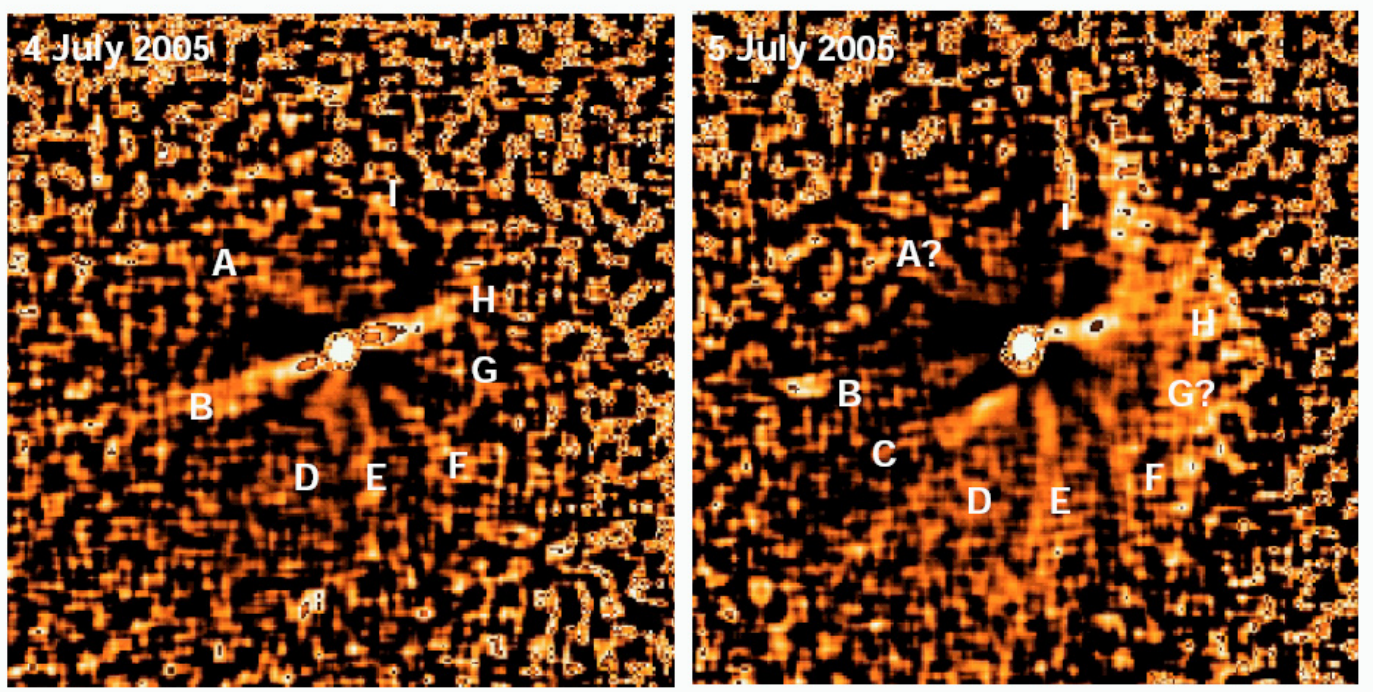

Fig. 1. Examples of Laplace-filtered images of 9P/Tempel 1, taken with FORS2 at the ESO VLT through I filter around 0UT on 4 and 5 July 2005. The coma structures, caused by normal activity and annotated A, B, C, D, E, F, G, H, I in the two subpanels, are also detectable when the ejecta cloud expanded throughout the coma. The rotation axis proposed by Thomas et al. (2006) and Lara et al. (2006) projects roughly in North-South direction about half way between structures D and E. The field of view is $40000 \times 40000 \mathrm{~km}$ at the distance of the comet. North is up, East to the left; Sun direction $\mathrm{PA}_{\text {sun }}=290 \mathrm{deg}$. The brightness peak in the coma (nucleus position) is in the center of the field of view. Noise fluctuations appear as random small-scale pixel areas in the processed images.

Table 3. Coma structure geometry caused by normal activity of comet 9P/Tempel 1 around the time of the DI event. Near-nucleus position angles PA, curvature and minimum extension are determined from BVRI images of the comet obtained between 4 and 8 July 2005. The results are cross-checked with data obtained through narrowband filters during the same time interval. The jet-like structures are labeled in Fig. 1. Accuracy of the PA values is \pm 5 deg. Active regions, tentatively associated with the jets, are labeled AR1, AR2, AR3a, AR3b, AR3c, and AR4. Abbreviations and notes: $\mathrm{C}=$ clockwise curvature, $\mathrm{CC}=$ counterclockwise curvature, $\mathrm{S}=$ straight, no curvature. $(*)$ Structure B may also be due to comet tail phenomena. (**) Structure C is detached from the nucleus, but overlaps with jet $\mathrm{D}$ at an projected nucleus distance of about $3500 \mathrm{~km}$. It extends almost parallel to structure B into tail direction. (***) Structures G, H, I evolved from the nucleus at about the same PA, but show different curvatures which indicates that they may originate from 3 different active regions at the nucleus with slightly different latitudes: $\mathrm{G}$ located slightly South of $\mathrm{H}$ and the latter being slightly South of I. The PA difference between GHI and the projected rotation axis suggests that the related active regions may be located close to the equator of the nucleus.

\begin{tabular}{ccccc}
\hline \hline Structure & $\begin{array}{c}\text { Nuc. PA } \\
(\mathrm{deg})\end{array}$ & Curvature & $\begin{array}{c}\text { Min. extension } \\
(\mathrm{km})\end{array}$ & $\begin{array}{c}\text { Active } \\
\text { Region }\end{array}$ \\
\hline $\mathrm{A}$ & 0 & $\mathrm{CC}$ & 27000 & $\mathrm{AR} 4$ \\
$\mathrm{~B}(*)$ & 110 & $\mathrm{~S}$ & 47000 & $\mathrm{AR3b}$ \\
$\mathrm{C}(* *)$ & - & $\mathrm{S}$ & 47000 & $\mathrm{AR2}$ \\
$\mathrm{D}$ & 155 & $\mathrm{C}$ & 58000 & $\mathrm{AR} 1$ \\
$\mathrm{E}$ & 195 & $\mathrm{C}$ & 43000 & $\mathrm{AR} 1$ \\
$\mathrm{~F}$ & 225 & $\mathrm{C}$ & 23000 & $\mathrm{AR2}$ \\
$\mathrm{G}(* * *)$ & 285 & $\mathrm{C}$ & 18000 & $\mathrm{AR3c}$ \\
$\mathrm{H}(* * *)$ & 285 & $\mathrm{~S}$ & 17000 & AR3b \\
$\mathrm{I}(* * *)$ & 285 & $\mathrm{CC}$ & 25000 & AR3a \\
\hline
\end{tabular}

our images of the first post-impact observing epoch about $17 \mathrm{~h}$ after the impactor hit the comet. At that time the ejecta cloud has expanded into the southwestern coma to a distance of about $14000 \mathrm{~km}$. Features A, B, D, E, F, G, H, and I remain visible also the following days when the ejecta cloud extended and attenuated further in the coma of the comet. This suggests that the

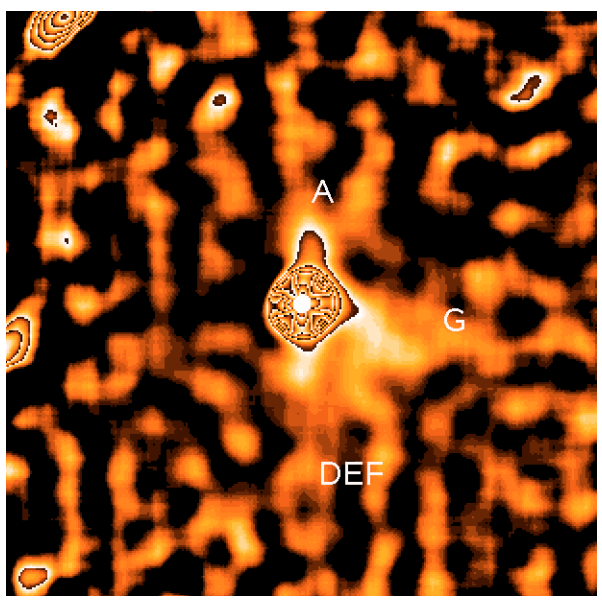

Fig. 2. $\mathrm{CN}$ filter image of $9 \mathrm{P} /$ Tempel 1 , taken with EMMI at the ESO NTT on 3 July 2005. Laplace filtering (see Sect. 2) is applied for structure enhancement. Structures A, DEF and G are identified, but not structure B. Field of view $=64000 \times 64000 \mathrm{~km}$ at the distance of the comet. North is up, East to the left; Sun direction $\mathrm{PA}_{\text {sun }}=290 \mathrm{deg}$.

ejecta material was optically thin by the time of our first postimpact imaging.

The pattern of jets gives the coma a porcupine-like appearance as previously observed in comet C/1995 O1 Hale-Bopp (Boehnhardt et al. 1997). The jets in the northern hemisphere of the coma show a counter-clockwise curvature, while the ones in the South turn clockwise into tail direction. They can be traced to at least $17000 \mathrm{~km}$ projected distance from the nucleus, some of them to more than $50000 \mathrm{~km}$ (Fig. 4). Compared to pre-impact monitoring results (Lara et al. 2006), the porcupine pattern appears to be relatively unchanged (only small shifts in the nearnucleus position angle and moderate changes of the curvature are noticeable). It is best interpreted as being caused by a system of embedded fans produced by several active regions on the rotating nucleus (Sekanina \& Boehnhardt 1997). During period 


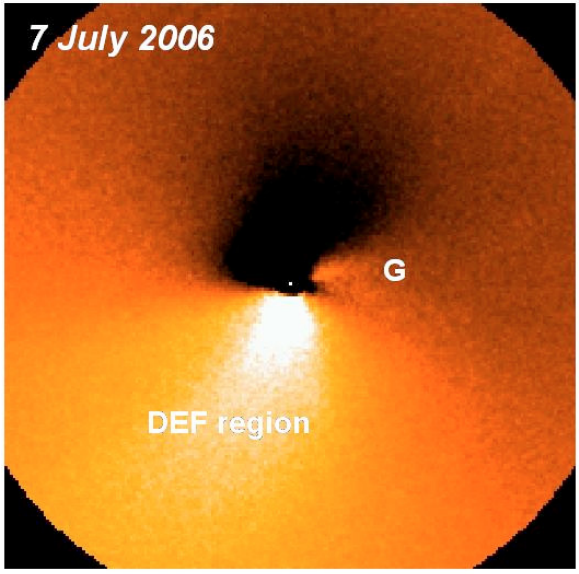

Fig. 3. Image of $9 \mathrm{P} /$ Tempel 1 , taken with FORS2 at the ESO VLT through $I$ filter around 23 UT on 7 July 2005, here processed by the radial renormalization method described in Sect. 2. Jet G is easily seen as is the southern fan which contains structures D, E, F. The field of view is $64000 \times 64000 \mathrm{~km}$ at the distance of the comet. North is up, East to the left; Sun direction $\mathrm{PA}_{\text {sun }}=290 \mathrm{deg}$. The dark segments in the image corners are due to masking and do not show science data.

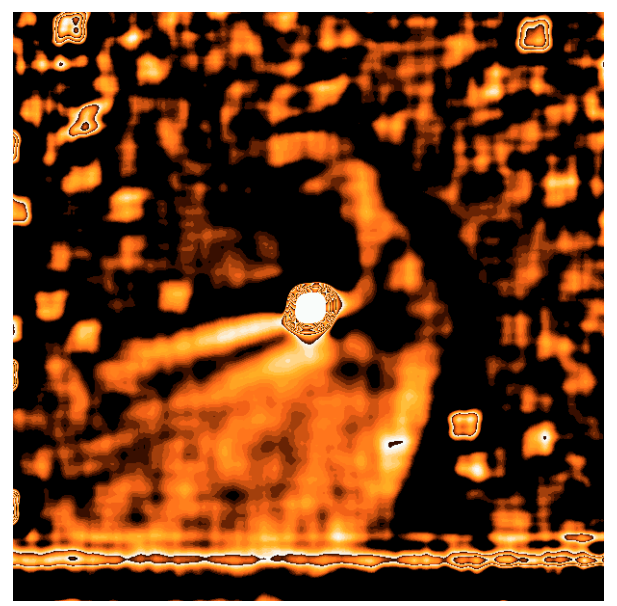

Fig. 4. Laplace-filtered image of 9P/Tempel 1, taken with FORS2 at the ESO VLT through $I$ filter around 23UT on 7 July 2005. Here a wider numerical filter is used to visualize the extension of the coma structures caused by normal activity of the comet. They extend between $17000 \mathrm{~km}$ (sunward direction at PA = $290 \mathrm{deg}$ ) to $58000 \mathrm{~km}$ (tailward direction at $\mathrm{PA}=110 \mathrm{deg}$ ). The field of view is $100000 \times 100000 \mathrm{~km}$ at the distance of the comet. North is up, East to the left; Sun direction $\mathrm{PA}_{\text {sun }}=290 \mathrm{deg}$. The brightness peak in the coma (nucleus position) is in the center of the field of view. The horizontal bars and noise structure South of the nucleus position is produced by the inter-chip gap of the FORS2 detector assembly.

2-10 July 2005 the direction of the nuclear rotation axis, as constrained by Lara et al. (2006) and more accurately determined from fly-by images by Thomas et al. (2006), projects approximately in North-South direction. The symmetric appearance of jets with respect to the orientation of the projected rotation axis suggests the assignment of active regions with the various coma features (see Table 3 ). We tentatively conclude that 4 surface areas may have been active (above the level of the overall cometary surface) around the time of the impact on 4 July 2005. One of this regions (AR3a-c in Table 3 ) may in fact be a multiple source at slightly different near-equatorial latitudes (producing structures $\mathrm{G}, \mathrm{H}, \mathrm{I})$.
The ejecta cloud: initially, the ejecta cloud expanded into the southwestern coma hemisphere (see Fig. 5). The main axis of the ejecta cone on 5 July 2005, i.e. about $21 \mathrm{~h}$ after impact, is found to be $235 \pm 5 \mathrm{deg}$. At the latest as of 6 July 2005 (but possibly earlier), part of the dust is pushed into tail direction by solar radiation pressure. On 8 July 2005 the ejecta cloud is still marginally detected above general coma background. By then, the ejecta dust extends about $25000 \mathrm{~km}$ into sunward direction and fills a large part of the southern, northern and eastern coma area. The base of the ejecta cone remains connected to the central brightness peak (=nucleus) in the coma at least up to 7 July 2005. The cloud has slightly higher surface brightness in the sunward half sector between PA about 230 to 300 deg (see Fig. 7) and it appears also slightly redder in the same region compared to the tailward half sector. These results are at least in qualitative agreement with expectations that the larger (thus "redder") dust remains longer in the sunward coma hemisphere than the smaller ("bluer") grains that are subject to higher radiation pressure.

By applying the simple fountain model (for a brief description see for instance Grün \& Jessberger 1990) to the motion of the ejecta grains, it is possible to estimate the initial velocity and radiation pressure factors $\beta$, i.e. the ratio between solar radiation pressure and solar gravity on the grains, using measurements of the apex distance of the ejecta cloud in sunward direction (i.e. at $\mathrm{PA}=290 \mathrm{deg}$ ). Here, the assumption is that all dust grains in the ejecta cloud are emitted at the same time (i.e. the impact time), but with different velocity and that at each observing epoch grains of a certain $\beta$ have reached the apex distance in solar direction at zero velocity before they turn around to be pushed into tail direction (or, in other words, the expansion of the ejecta dust in solar direction can be viewed as a "mass spectrometer" for the dust grains). The latter situation is certainly true for the front edge of the dust cloud on 6, 7 and 8 July 2005 and may be close to be fulfilled for 5 July 2005 as well, although there are no evident indications for dust being pushed into tailward direction in our images of this day. Table 4 lists the results for the initial velocity versus solar radiation factor $\beta$ of the ejecta dust. The range of $\beta$ and velocity is from 1.9 and $370 \mathrm{~m} \mathrm{~s}^{-1}$ to 0.2 and $160 \mathrm{~m} \mathrm{~s}^{-1}$. The beta ratios compare (see Grün \& Jessberger 1990 ) to dust grains of density $\sim 0.5 \mathrm{~g} / \mathrm{cm}^{3}$ and $0.8-6 \mu \mathrm{m}$ radius for absorbing materials. High $\beta$ values of 1.9 measured for the dust apex distance on 5 July 2005, are unlikely for dielectric grains. However, these type of grains can reach the apex distances measured as of 6 July 2005 if the grain radius is between $1.5-4 \mu \mathrm{m}$. The results suggest that the majority of the dust ejecta seen in the visible wavelength range consisted of grains of a few $\mu \mathrm{m}$ in size.

In our images of 7 July 2005 a small brightness enhancement is seen in the center of the coma (see Fig. 6). The diffuse cloud extends about $3500 \mathrm{~km}$ into the southwestern coma sector (between PA 180-250 deg). It is less apparent on 8 July 2005. From the available images it is not possible to conclude whether this cloud is an aftermath activity from the impact area on the nucleus (less likely since the impact region was not illuminated by the Sun) or it reflects stronger dust activity from one of the localized active regions that are connected to the fan structures in this part of the coma.

\section{Gas production rates}

Useful measurements of gas production rates of $9 \mathrm{P}$ are obtained for 3, 5, 9 and 10 July 2005 and of the dust reddening slope for 3, 9 and 10 July 2005 (see Table 5). $\mathrm{CN}, \mathrm{C}_{3}$ and $\mathrm{C}_{3}$ gas emission is detected, while $\mathrm{Na}$ and $\mathrm{NH}_{2}$ gas emission could not be 

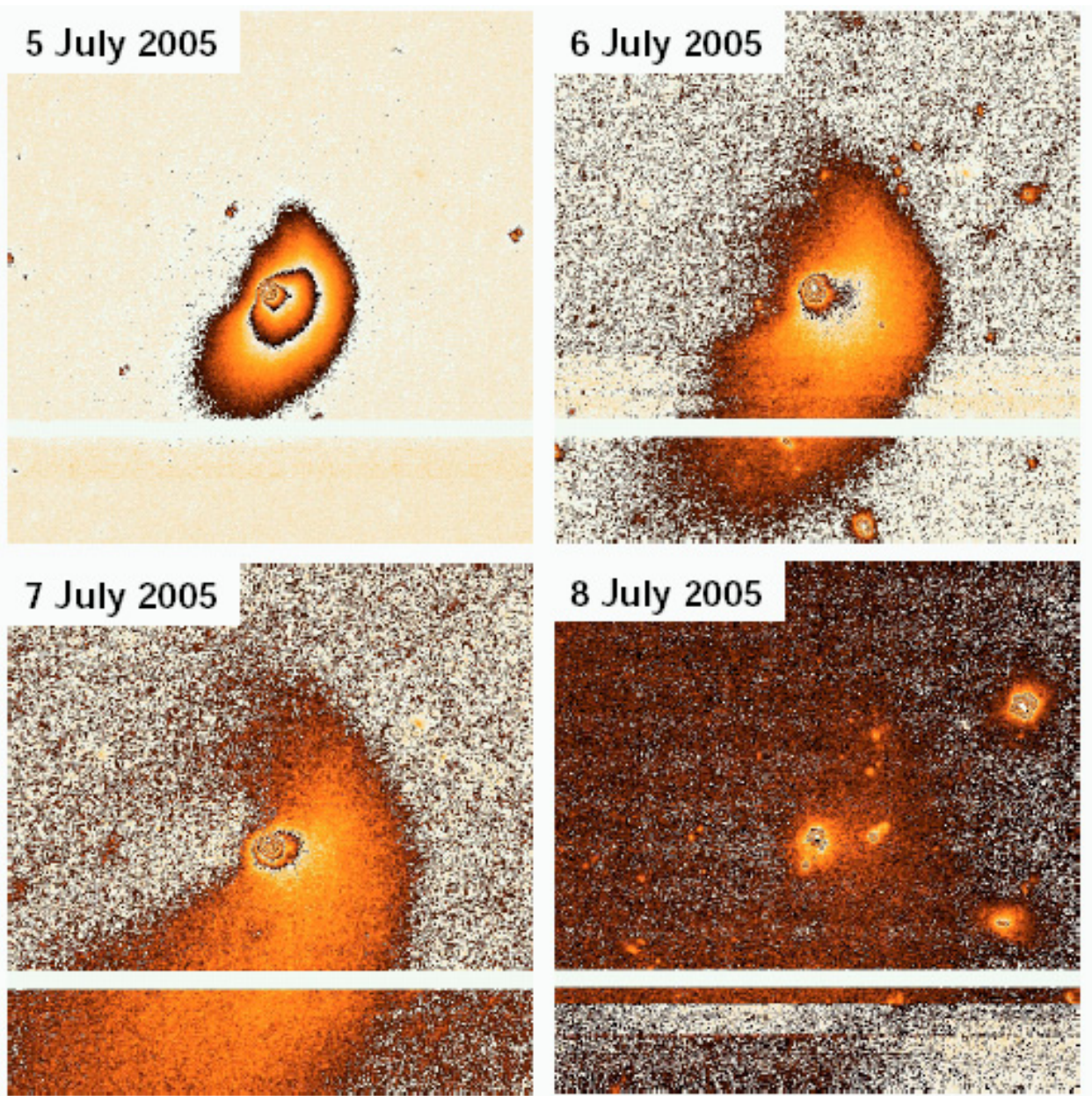

Fig. 5. I filter images of the dust ejecta cloud produced by DI. The sub-panels show the isophote pattern of the expanding cloud on 5, 6, 7, and 8 July 2005 around 0UT. Enhancement method 3 described in Sect. 2 is applied to remove the coma background from normal activity of the comet. The field of view is $87000 \times 87000 \mathrm{~km}$ at the distance of the comet. North is up, East to the left; sun direction $\mathrm{PA}_{\text {sun }}=290$ deg. The brightness peak in the coma (nucleus position) is in the center of the field of view. The horizontal bars and noise structure South of the nucleus position is due to the inter-chip gap of the FORS2 detector assembly.

Table 4. The DI dust cloud "mass spectrometer": Apex distance, radiation pressure factor $\beta$ and initial velocity as determined from the DI dust ejecta envelope.

\begin{tabular}{lcccc}
\hline \hline $\begin{array}{l}\text { Date/time } \\
\text { (UT) }\end{array}$ & $\begin{array}{c}\text { Time after } \\
\text { impact }(\mathrm{h})\end{array}$ & $\begin{array}{c}\text { Apex distance } \\
(\mathrm{km})\end{array}$ & $\beta$ & $\begin{array}{c}\text { Velocity } \\
\left(\mathrm{m} \mathrm{s}^{-1}\right)\end{array}$ \\
\hline $05-07-2005 / 02: 45$ & 20.9 & 14100 & 1.9 & 370 \\
$06-07-2005 / 00: 00$ & 32.0 & 19400 & 0.65 & 260 \\
$07-07-2005 / 00: 00$ & 66.1 & 22900 & 0.3 & 190 \\
$07-07-2005 / 23: 00$ & 89.2 & 25000 & 0.2 & 160 \\
\hline
\end{tabular}

measured above the background of the dust continuum. The OII filter images had not enough flux at larger nuclear distances to be useful for determining the reddening slope in the blue-to-UV wavelength region.

CN: the production rates of the $\mathrm{CN}$ molecules one day before ( 3 July) and 5 to 6 days after the DI event are almost unchanged. They are systematically lower than those of Schleicher et al. (2006) and Rauer et al. (2006), who used slightly different Haser model parameters than us, but at the same level than those of Lara et al. (2007) who applied the same parameters as we did. A small decrease of $\mathrm{Q}(\mathrm{CN})$ during the observing interval is suggested by our measurements, which would be in-line with a decay of similar amplitude as measured by Schleicher et al.

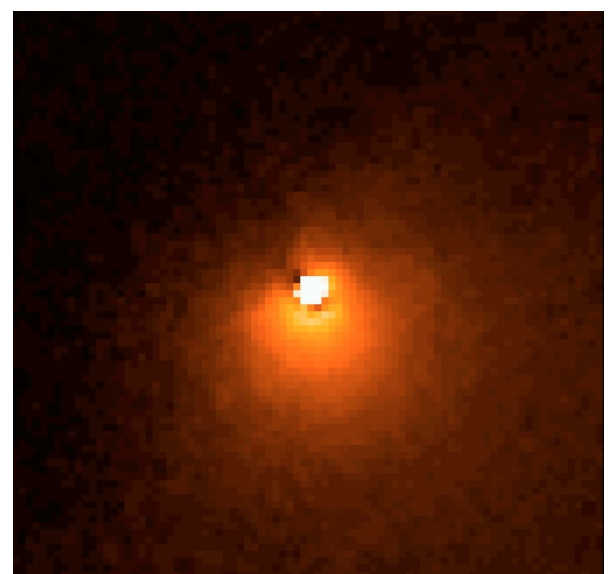

Fig. 6. $R$ filter images of the DI dust ejecta cloud on 7 July 2005. The image shows a moderate dust activity in the southwestern coma quadrant. Image processing and image orientation as for Fig. 5, the field of view is $14000 \times 14000 \mathrm{~km}$ at the distance of the comet.

However, in both cases the change in the production rate is at a marginal level.

$\mathbf{C}_{3}$ : our measurements of the production rates of $\mathrm{C}_{3}$ molecules are at a low signal level only. They may hence be 
Table 5. Fluxes, molecule production rate\&production rate ratios and dust continuum reddening of 9P/Tempel 1 during 2-10 July 2005 . The fluxes $\mathrm{F}$ and reddening slopes $R$ are measured in circular apertures of fixed radii centered at the brightness peak in the coma. $Q_{\text {mean }}$ and $R_{\text {mean }}$ are mean values of the production rates and reddening slopes, respectively, obtained from the flux and slope measurements with radii 5000, 10000, 15000, and $20000 \mathrm{~km}$. The continuum filter image used for dust continuum subtraction in the gas filter images is indicated in table column DCONT; if two filters are given, the flux and production rate numbers are the mean values obtained from measurements using either of the two filter images as reference for the dust continuum subtraction. The table lists logarithmized values for fluxes and production rates. "NM" means "no useful measurement obtained". Flux $F$ is measured in erg/s/ $/ \mathrm{cm}^{2} / \AA ̊ \AA$, production rates $Q$ in molecules/s, reddening slope $R$ in $\% / 100 \mathrm{~nm}$, aperture radii are in $\mathrm{km}$ at the distance of the comet. All results are based on narrowband filter imaging of the comet obtained with the EMMI instrument at the ESO NTT telescope in La Silla. Measurements, obtained at very high airmass where the flux calibration may be badly affected by extinction, are indicated by symbol *.

\begin{tabular}{|c|c|c|c|c|c|c|c|}
\hline \multirow[t]{2}{*}{ Gas component } & \multirow[t]{2}{*}{ Date } & \multicolumn{4}{|c|}{$\log (F)$ in aperture of radius } & \multirow[t]{2}{*}{$\overline{\mathrm{DCONT}}$} & \multirow[t]{2}{*}{$\overline{\log (Q)}$} \\
\hline & & 5000 & 10000 & 15000 & 20000 & & \\
\hline \multirow[t]{3}{*}{$\mathrm{CN}$} & 2.99 & -14.087 & -13.550 & -13.263 & -13.071 & $\mathrm{BC}, \mathrm{RC}$ & $24.84 \pm 0.1$ \\
\hline & 9.15 & -13.693 & -13.393 & -13.193 & -12.925 & $\mathrm{BC}, \mathrm{RC}$ & $24.73 \pm 0.1$ \\
\hline & 10.05 & -13.747 & -13.438 & -13.232 & -12.962 & $\mathrm{BC}, \mathrm{RC}$ & $24.68 \pm 0.1$ \\
\hline \multirow[t]{2}{*}{$\mathrm{C}_{3}$} & 3.01 & -14.651 & -14.133 & -13.872 & -13.706 & $\mathrm{BC}, \mathrm{RC}$ & $23.57 \pm 0.2$ \\
\hline & 10.10 & -14.657 & -14.142 & -13.873 & -13.698 & $\mathrm{BC}$ & $24.18 \pm 0.2$ \\
\hline \multirow[t]{4}{*}{$\mathrm{C}_{2}$} & 3.04 & -14.450 & -13.884 & -13.572 & -13.363 & $\mathrm{BC}, \mathrm{RC}$ & $24.84 \pm 0.1$ \\
\hline & 5.18 & NM & NM & -14.257 & -13.832 & $\mathrm{RC}$ & $24.30 \pm 0.2 *$ \\
\hline & 9.18 & -14.804 & -14.197 & -13.869 & -13.655 & $\mathrm{BC}$ & $24.55 \pm 0.1$ \\
\hline & 9.98 & -14.452 & -13.945 & -13.682 & -13.385 & $\mathrm{BC}, \mathrm{RC}$ & $24.90 \pm 0.15$ \\
\hline \multirow[t]{2}{*}{ Dust } & Date & \multicolumn{4}{|c|}{$R$ in aperture of radius } & DCONT & $R_{\text {mean }}$ \\
\hline & & 5000 & 10000 & 15000 & 20000 & & \\
\hline \multirow[t]{3}{*}{ Dust reddening } & 3.06 & 8.6 & 9.2 & 9.3 & 9.1 & $\mathrm{BC}+\mathrm{RC}$ & $9.0 \pm 0.5$ \\
\hline & 9.20 & 16.1 & 18.4 & 20.4 & 22.1 & $\mathrm{BC}+\mathrm{RC}$ & $19.3 \pm 3$ \\
\hline & 10.01 & 17.5 & 19.4 & 21.3 & 22.9 & $\mathrm{BC}+\mathrm{RC}$ & $20.3 \pm 3$ \\
\hline
\end{tabular}
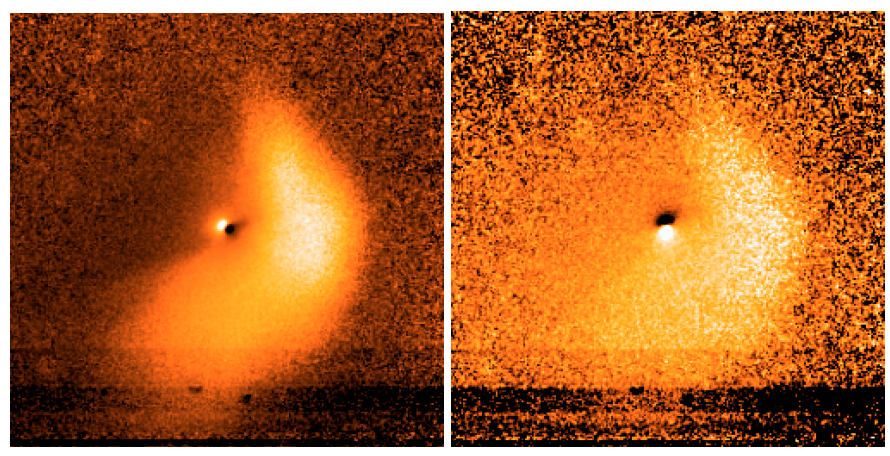

Fig. 7. $R$ filter intensity (left) and $R-V$ color (right) of the DI dust ejecta cloud on 5 July 2005. The surface brightness is higher in the sunward sector compared to the tailward side (brighter region in left panel). The sunward cloud sector is also redder than the tailward one (brighter region in right panel). Image processing and image orientation as for Fig. 5, the field of view is $43000 \times 43000 \mathrm{~km}$ at the distance of the comet.

affected by larger errors than obtained from the standard error analysis (as indicated in Table 5). As a consequence the increase of the $\mathrm{C}_{3}$ production between 3 and 10 July as is suggested by our measurements, should be taken with care. Our results are clearly below the values of Schleicher et al. (2006), and are in better agreement with those of Lara et al. (2007).

$\mathbf{C}_{2}$ : the production of $\mathrm{C}_{2}$ molecules is at a very similar level on 3 and 10 July 2005. The drop on 5 July 2005 may be due to extinction since the observations were taken at very high airmass and no extinction values could be determined for this night. It is noteworthy, however, that a similarly low production rate of $\mathrm{C}_{2}$ is measured on 9 July 2005, when the sky conditions were good and the observations were taken at lower airmass. With the exception of 5 and 9 July, our results are in agreement with those of Schleicher et al. (2006) and Lara et al. (2007).
Production rate ratios: we determined ratios of molecule production as follows: $\log \left(\mathrm{C}_{3} / \mathrm{CN}\right)$ from -1.3 to -0.5 , $\log \left(\mathrm{C}_{2} / \mathrm{CN}\right)$ from -0.2 to 0.2 and $\log \left(\mathrm{C}_{3} / \mathrm{C}_{2}\right)$ from -0.9 to -1.3 . Following the characterization scheme of A'Hearn et al. (1995), our results indicate that $9 \mathrm{P} / \mathrm{Tempel} 1$ is a typical comet concerning the mixture of $\mathrm{C}_{3} / \mathrm{C}_{2} / \mathrm{CN}$ gas producing material. Our values for the production rate ratios are in good agreement with the results of Lara et al. $(2006,2007)$ for the impact and pre-impact periods, but they obviously differ to those of Schleicher et al. (2006) for the $C_{3}$ molecules.

\section{Dust reddening}

There are two main features seen in our results for the reddening slope of the dust coma of 9P/Tempel 1: (1) the reddening changes from about $9 \% / 100 \mathrm{~nm}$ on 3 July 2005 to about $20 \% / 100 \mathrm{~nm}$ on 9 and 10 July 2005; (2) the reddening was rather constant through out the measured coma on 3 July 2005, while it showed increasing slope with nuclear distance on 9 and 10 July 2006. These changes in the dust reddening from before to after impact could be a consequence of the DI event. In that case one has to assume that the dust colors changed on a global scale due to the impact event, and that this change persisted despite the fact that the dust ejecta cloud was very much dispersed 4 days after the impact and almost no longer detectable in direct images of the comet. Similar absolute values and a comparable change in the dust reddening slope are also reported in the Calar Alto spectroscopy of the comet (Lara et al. 2007). According to their measurements the change happened from 5 to 6 July 2005 and, according to our data, it may have persisted at about constant level for at least 4 days longer. The increasing reddening with radial distance could at least qualitatively be understood in a scenario like: the dust ejecta expanded into the coma and by about 2 days after impact they filled most of our coma aperture. Since they are redder than the dust from normal activity, the reddening slope changed to higher values, although close to the nucleus new dust from normal activity evolved, hence reducing the dust 
reddening slope systematically for closer distances to the coma center.

\section{Conclusions}

Around the time of the DI event, comet 9P/Tempel 1 showed a twofold coma pattern:

- the normal activity of the comet displayed a porcupine pattern of embedded coma fans produced by at least 4 active regions on the rotating nucleus. This activity remained undisturbed by the DI event. The production rates of the minor species $\left(\mathrm{CN}, \mathrm{C}_{3}, \mathrm{C}_{2}\right)$ on $9+10$ July 2005 is widely unchanged compared to the pre-impact situation. Before impact the dust reddening was uniform within $20000 \mathrm{~km}$ distance from the nucleus at a $9 \% / 100 \mathrm{~nm}$ level.

- the dust ejecta cloud is detectable in our images between 5 and 8 July. The visible image of the cloud is dominated by $\mu \mathrm{m}$-size grains that expanded into the coma at a speed of a few $100 \mathrm{~m} \mathrm{~s}^{-1}$. Absorbing material can best explain the measured $\beta$ range; however, dielectric grains may also exist among the ejecta dust. The ejecta cloud was optically thin $21 \mathrm{~h}$ after impact. The surface brightness was higher and the dust color was redder in the sunward sector of the cloud. The reddening slope of the overall coma was affected by the DI ejecta: the mean value is about $20 \% / 100 \mathrm{~nm}$ and an increase with radial distance is found.

Acknowledgements. We like to thank in the first place the scientific, technical and administrative staff of ESO Chile and Garching who were involved in organizing and performing together with our ESO DI dust team this complex and demanding observing campaign at several ESO telescopes of the two observatories La Silla and VLT Paranal. We like to acknowledge the National Observatory of Bulgaria for providing us with a sodium filter for the observing campaign at the NTT telescope in La Silla. Luisa Lara from the Instituto Astrofisico de Andalucia in Granada/Spain was very helpful in the interpretation of our results by providing information from own observations of the DI event obtained at the Calar Alto Observatory in Spain. We also wish to thank Mike A'Hearn, principle investigator of the DI mission from the University of Maryland, USA, and Karen Meech, coordinator of the World Observatory campaign of the event from the University of Hawaii, USA, for their support of our program and for providing own unpublished DI mission results that helped interpreting our own measurements.

\section{References}

A'Hearn, M. F., \& Vanysek, V. 1985, IHW Newsletter - Photometry and Polarimetry Net, issue 8 Nov. 1985

A'Hearn, M. F., Hoban, S., Birch, P. V., et al. 1986, Nature, 324, 649

A'Hearn, M. F., Millis, R. L., Schleicher, D. G., et al. 1995, Icarus, 118, 223

A'Hearn, M. F., Belton, M. J. S., Delamere, A., et al. 2005, SSRv, 117, 1

Boehnhardt, H., \& Birkle, K. 1994, A\&AS, 107, 101

Boehnhardt, H., Birkle, K., Fiedler, A., et al. 1997, EMP, 78, 179

Bonev, T., Ageorges, N., Boehnhardt, H., et al. 2007 [arXiv:astro-ph/0703554]

Hamuy, M., Suntzeff, N. B., Hezthcote, S. R., et al. 1994, PASP, 106, 566

Grün, E., \& Jessberger, E. K. 1990, Dust, in "Physics and Chemistry of Comets", ed. W. F. Huebner (Heidelberg: Springer Press), 113

Haser, L. 1957, Cl. Sci. Acad. R. Belg., 43, 740

Jehin, E., Manfroid, J., Hutsemekers, D., et al. 2006, ApJ, L145

Käufl, H. U., Ageorges, N., Bagnulo, S., et al. 2005, The Messenger, 121, 11

Küppers, M., Bertini, I., Fornasier, S., et al., 2005, Nature, 437, 987

Landolt, A. U. 1992, AJ, 104, 372

Lara, L., Boehnhardt, H., Gredel, R., et al. 2006, A\&A, 445, 1151

Lara, L., Boehnhardt, H., Gredel, R., et al. 2007, A\&A, 465, 1061

Meech, K., Ageorges, N., A'Hearn, M. K., et al. 2005, Science, 310, 265

Murtagh, F., Zeilinger, W. W., Starck, J. L., \& Boehnhardt, H. 1993, The Messenger, 73, 37

Rauer, H., Weiler, M., Sterken, C., et al. 2006, A\&A, 459, 257

Schleicher, D. G. The fluorescence of cometary CN and OH, Ph.D. Thesis, Univ. Maryland, College Park

Schleicher, D. G., Barnes, K. L., \& Baugh, N. F. 2006, AJ, 131, 1130

Sekanina, Z. 1987, ESA-SP, 278, 315

Sekanina, Z., \& Boehnhardt, H. 1997, EMP, 78, 313

Schwarz, G., Cosmovici, C. B., Crippa, R., et al. 1997, EMP, 78, 189

Thomas, P. C., A'Hearn, M. F., et al. 2006, Icarus, 187, 4

Tozzi, G. P., Boehnhardt, H., Kolokolova, L., et al. 2007, A\&A, submitted 